\title{
Thermal buckling and post-buckling improvements of laminated composite plates using finite element method.
}

\begin{abstract}
Thermal buckling and thermal post-buckling behaviours of laminated composite plates are improved by embedding shape memory alloy wires within laminates of composite plates. The procedure is to use the recovery stress that is induced when the reverse transformation of the shape memory alloy from martensite to austenite phases is constrained. For aerospace applications where the source of the shape memory alloy heating is the high temperature environment itself, a study is conducted to see the effect of shape memory alloy in improving the thermal buckling and post-buckling of composite plates. Due to the temperature dependent nature of the composite matrix and the shape memory alloy, the finite element formulation developed here is in the incremental form. Solving this non-linear model using the developed in-house source code, critical loads are determined and the post-buckling paths of the shape memory alloy composite plates are traced. This study shows that by embedding the shape memory alloy within composite plates, the thermal buckling and post-buckling behaviours of composite plates can be improved substantially.
\end{abstract}

